\title{
A comparison of the effects of extinction and satiety on operant response duration in the rat
}

\author{
LOWELL T. CROW \\ Western Washington University, Bellingham, Washington 98225
}

\begin{abstract}
Rats were maintained on a 23.5-h water deprivation schedule and trained in CRF operant responding for water. During the daily .5-h operant session, measures were made of response duration at successive 1-min intervals under both reinforcement and extinction conditions, and comparisons were drawn between the following groupings of the data: (1) reinforcement for the first $5 \mathrm{~min}$ of responding, (2) reinforcement for the last 5 min of responding, (3) extinction for the first $5 \mathrm{~min}$ of responding, (4) extinction for the last $5 \mathrm{~min}$ of responding. The results were that both extinction and satiety increased the mean and variability of response duration such that grouping $4>3,2>1$. The results are believed to support a common variability hypothesis for both kinds of behavioral change.
\end{abstract}

Response duration in operant responding decreases in both mean and variance with continuous reinforcement contengencies and increases in mean and variance with extinction (Hurwitz, 1954; Schwartz \& Williams, 1972). Hurwitz (1954) also noted these effects with "extended rewarded practice," an effect he attributed to either fatigue or a reduction in motivation. Subsequent work by Crow, Coop, and Carlock (1977) and Crow, Westveer, and Kass (1976) indicated that the increases in mean and variance of leverpressing duration associated with such extended rewarded practice were not fatigue-induced, but due to satiation.

That satiety is closely related to processes of extinction, and that there may be "resistance to satiation" analogous to resistance to extinction has been pointed out previously (Morgan, 1974), and it is possible that the similarity in response duration change occasioned by both extinction and satiety is due to a common element. It has been proposed by the present author (Crow, 1977) that behavioral variability is proportional to the tolerance of the environment to behavioral alteration, and the question arose as to the relevance of this hypothesis to the increased variance seen in satiety and extinction. If both kinds of variance changes are due to common ultimate effects of tolerance changes, a direct comparison of response duration variances should reveal a specific ordinal relation between situations in which either one or both effects were present.

\section{METHOD}

\section{Subjects}

Sixteen female rats 100 to 150 days of age were used. The animals were taken from a basic Long-Evans strain from the Western Washington University colony.

\section{Apparatus}

The apparatus has been described elsewhere (Crow et al., 1976). It consisted of four water-delivery operant chambers equipped to measure response duration of leverpressing at any interval within a drinking session.

\section{Procedure}

All animals were adapted to a $23.5-\mathrm{h}$ water deprivation schedule and trained to a stable level of leverpressing before measures of satiation were begun. After a stable level of response duration had been attained, measures were made of number of responses and average response duration for each minute of the 30-min leverpressing (drinking) session. The first and last five 1-min segments in which responding occurred were used to obtaind a "reinforced thirst" and a "reinforced satiety" condition, respectively.

The extinction data were then collected by extinguishing three 1-min segments of the total 30 -min session on each day until all of the $30-\mathrm{min}$ data for each animal had been accumulated. The 1-min segments for a given day were chosen to represent the initial, middle, or concluding periods of the entire session, but such that there would be no consecutive 2 min of extinction on any day. Also, measures were made on alternate days so that a full $30 \mathrm{~min}$ of reinforcement always followed the day on which extinction data were taken. The extinction part of the study thus required 20 days. From these extinction data were taken (as above) an "extinction thirst" and an "extinction satiety" condition.

\section{RESULTS}

Figure 1 shows the effects upon mean response duration of the two conditions, extinction and satiety. By sign test (Siegel, 1956) all points differed significantly at the .05 confidence level. That is, an increase in response duration accompanied those leverpresses in the latter $5 \mathrm{~min}$ vs. the initial $5 \mathrm{~min}$ of responding within the 30-min drinking session, and this increase in response duration as thirst was sated occurred under both reinforcement and extinction conditions. In support of a satiety interpretation of these differences, the mean number of responses decreased from the initial to concluding segments of the session in extinction and reinforcement conditions. The number of responses from the first to the last 5-min responding segments dropped 


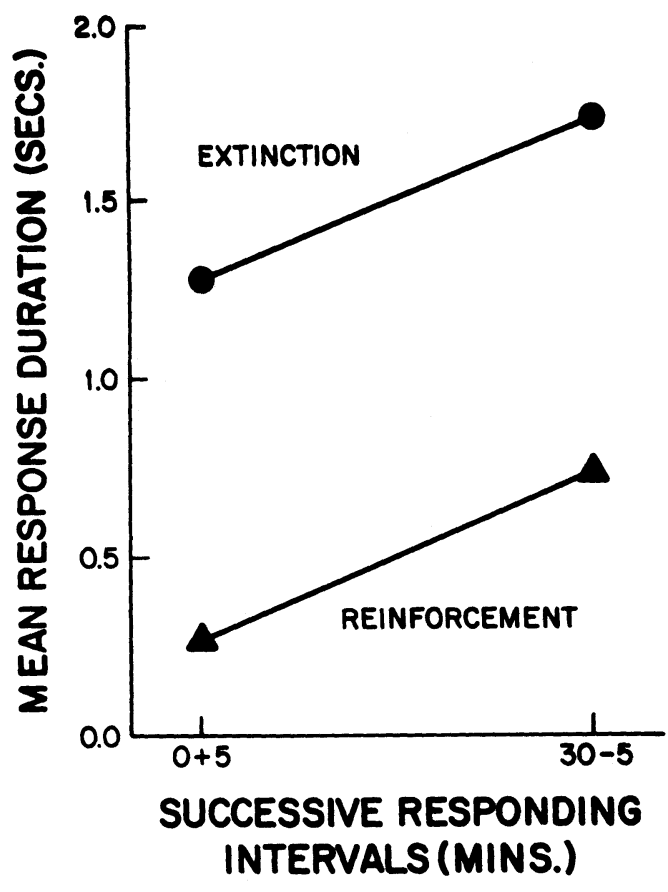

Figure 1. Mean response duration for the initial (thirst) and concluding (satiety) segments of the drinking period. Each point represents 16 animals.

from 9.96 to 3.20 in the extinction situation and from 7.9 to 2.4 in the reinforcement condition. Both of these changes are significant at the .05 level by sign test.

Figure 2 shows the changes in response duration variance corresponding to those changes in mean response duration shown in Figure 1. By sign test there was a significant increase in variance from the first to the last 5-min responding segments in the case of the reinforcement condition $(p<.01)$ and in the case of the extinction condition $(\mathrm{p}=.011)$. There was a significant difference between the reinforcement and extinction conditions at the initial 5-min responding segment $(p<.01)$ and a significant difference at the concluding 5 -min responding segment $(p=.002)$. There was no significant difference between the response duration variances of the initial 5-min extinction phase and the concluding 5 -min segment of the reinforcement phase $(\mathrm{p}=.402)$.

\section{DISCUSSION}

The results support the findings of Hurwitz (1954) and Schwartz and Williams (1972) of an increase in both mean and variance of response duration in extinction, and the findings by Crow et al. (1977) and Crow et al. (1976) of an increase in both mean and variance of response duration in satiety. In addition, the findings are believed to support the notion of a commonality of elements in the extinction and satiety processes (Morgan, 1974).

Figure 3 summarizes the variance findings in correspondence with a model of behavioral variability proposed previously (Crow, 1977).

If tolerance factors for variation are assumed for both the extinction and satiety conditions, and if these factors are cumulative in their behavioral effects, the ordinal relation of the variances that were found for each condition would be expected. That is, greater tolerance as a unitary concept resulted in greater behavioral variability. The variance values closely approximate a log progression with successive increases in tolerance and are reminiscent of a multiplicative relationship of drive and habit

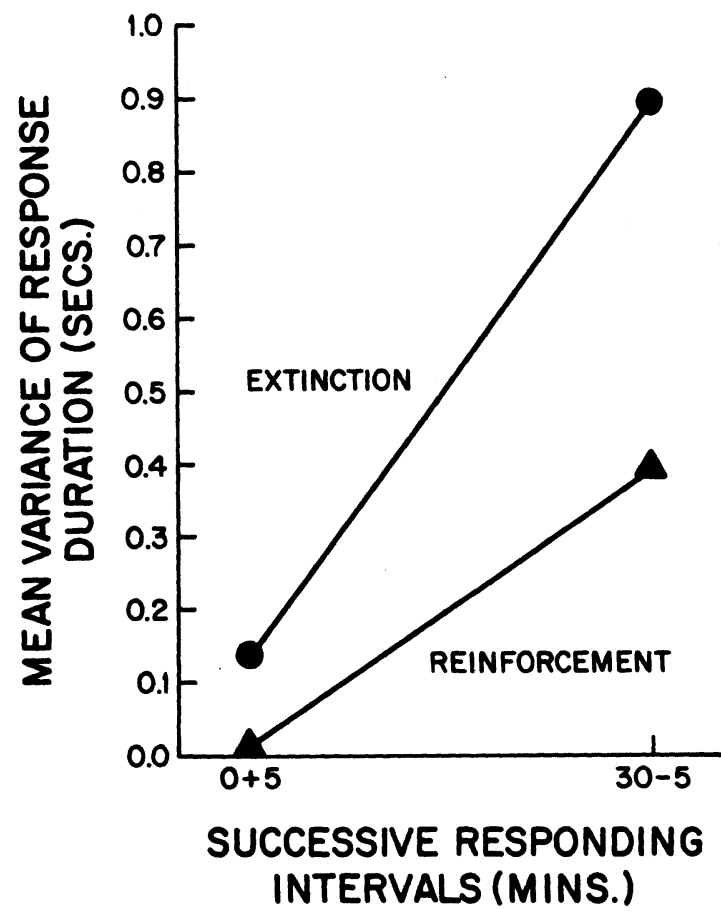

Figure 2. Mean variance of response duration for the initial (thirst) and concluding (satiety) segments of the drinking period. Each point represents 16 animals.

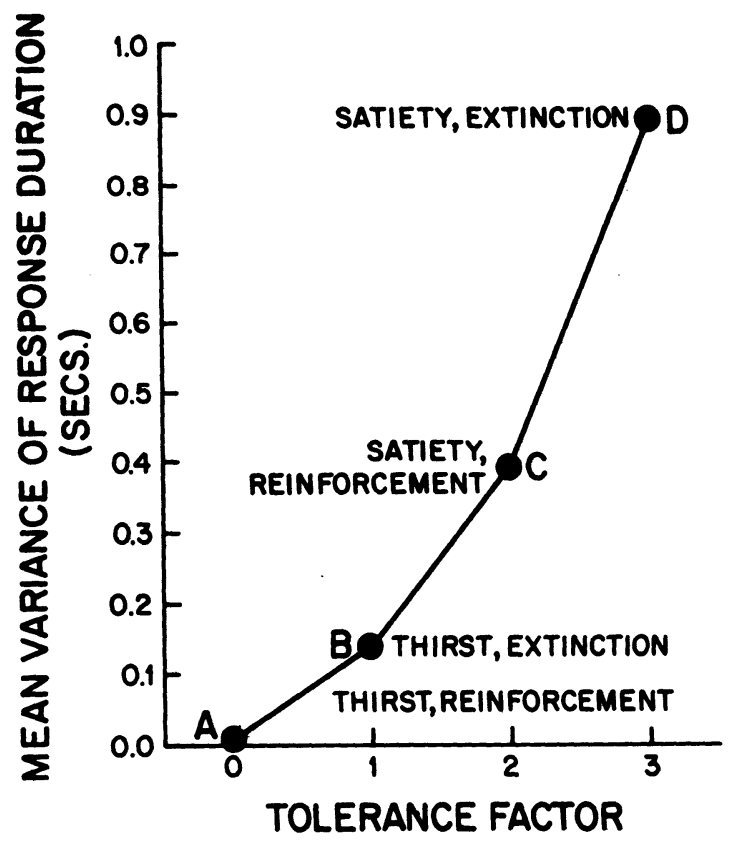

Figure 3. Behavioral variability as a function of prevailing tolerance factors. 
factors. The conditions giving rise to the changes occurring in extinction and satiety certainly differ markedly, but the ultimate central nervous mechanisms of such behavioral alteration may be one and the same process and subject to a more unified theoretical treatment than previously allowed.

\section{REFERENCES}

CRow, L. Is variability a unifying behavioral concept? Psychological Record, 1977, 27, 783-790.

Crow, L.. Coop, B., \& Carlock, L. Satiety-dependent microbehavior in water ingestion by the rat: The effects of salt and water preloads on response duration. Bulletin of the Psychonomic Society, 1977, 9, 349-352.
Crow, L., Westveer, J., \& Kass, R. Satiety-dependent microbehavior in water ingestion by the rat: The effects of alcohol on response duration. Physiological Psychology, 1976, 4, 163-165.

HuRwitz, H. Response duration of lever pressing in the rat. Quarterly Journal of Experimental Psychology, 1954, 6, 62-71.

Morgan, M. Resistance to satiation. Animal Behavior, 1974. 22. 449-466.

Schwartz, B.. \& Williams, D. Two different kinds of key peck in the pigeon: Some properties of response maintained by negative and positive response-reinforcer contingencies. Journal of the Experimental Analysis of Behavior, 1972, 18, 201-216.

SIEGEL, S. Nonparametric statistics for the behavioral scientist. New York: McGraw-Hill, 1956.

(Received for publication October 14, 1977.) 